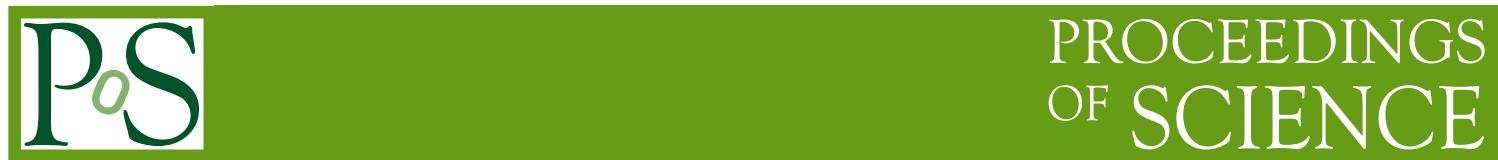

\title{
Searches for Hadronic Resonances at CMS
}

\section{David Yu Y† $^{* \dagger}$}

Brown University

E-mail: david yudbrown.edu

New particles decaying to jet pairs, predicted by many theories of physics beyond the Standard Model, could be discovered at the Large Hadron Collider as resonances in the dijet invariant mass spectrum. Three searches for dijet resonances by the CMS Collaboration are presented, which employ several novel techniques to probe a wide range of resonance masses. The first search targets resonance masses above $1800 \mathrm{GeV}$. A new background method using jet pairs with large pseudorapidity separation is described. The latter two searches target resonances below $450 \mathrm{GeV}$, where trigger bandwidth limitation preclude the methods used at high masses. The resonances are required to be produced with high transverse momentum due to significant initial state radiation (ISR), and hence the resonance decay products are collimated into a single jet. The searches, which require either ISR jets or photons, probe resonance masses from $50 \mathrm{GeV}-450 \mathrm{GeV}$ and $10 \mathrm{GeV}-125 \mathrm{GeV}$, respectively.

7th Annual Conference on Large Hadron Collider Physics - LHCP2019

20-25 May, 2019

Puebla, Mexico

\footnotetext{
* Speaker.

†n behalf of the CMS collaboration
} 


\section{Introduction}

New hadronic resonances are predicted by many theories of physics beyond the Standard Model (SM). New particles such as additional gauge bosons [1, 2], excited quarks [3, 4], or RandallSundrum gravitons [5] could be discovered at hadron colliders as resonances in the dijet invariant mass spectrum. Searches for such resonances thus have a long tradition at hadron colliders, including the Large Hadron Collider (LHC) [6]. At the LHC, however, the ever-increasing instantaneous luminosity and the corresponding increases in trigger thresholds have pushed the mass range for traditional dijet searches to well above $1 \mathrm{TeV}[7,8]$. Several new techniques have been developed to maintain sensitivity to lower mass resonances. During Run 1, the use of trigger-level data, i.e. the jets reconstructed by the high-level trigger algorithms, enabled resonance masses as low as $450 \mathrm{GeV}$ to be probed $[9,10]$. Triggers with online $\mathrm{b}$ tagging have probed as low as $325 \mathrm{GeV}$ [11]. More recently, searches for resonances produced with large initial state radiation (ISR) have probed as low as $10 \mathrm{GeV}$.

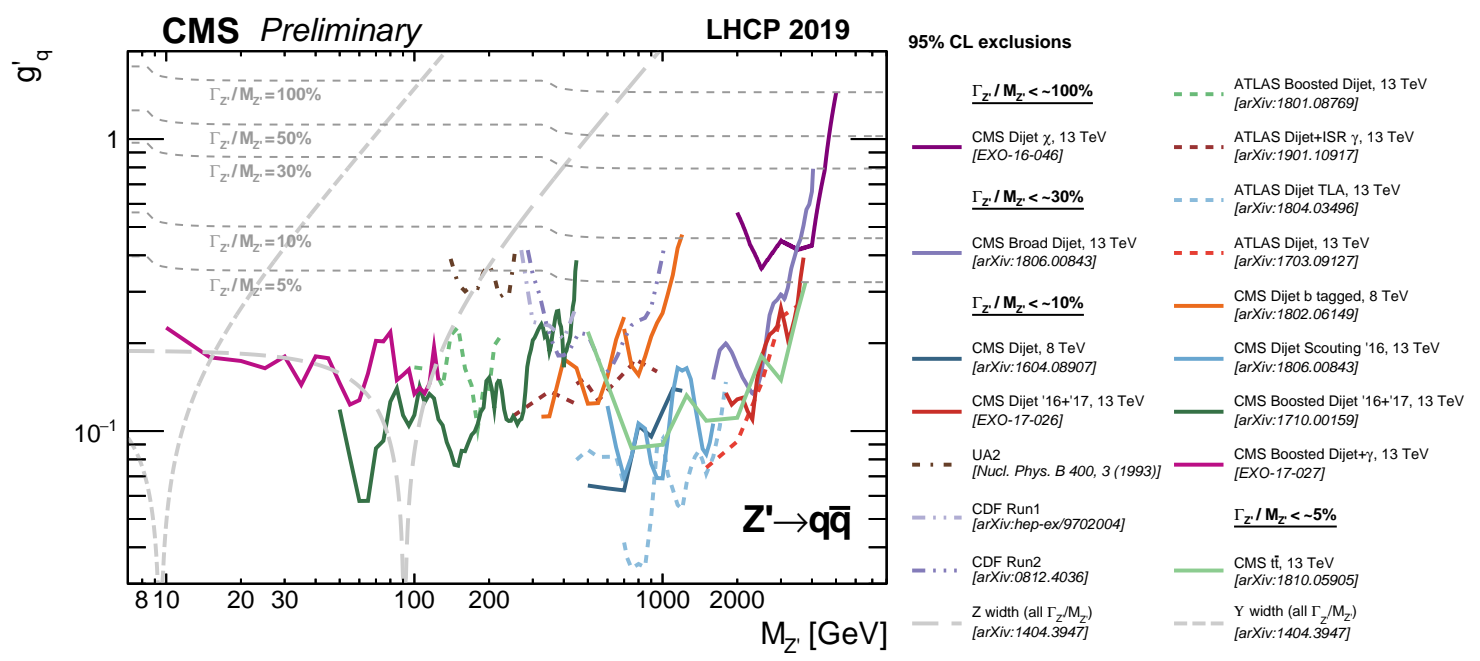

Figure 1: Limits on the universal coupling $g_{q}^{\prime}$ between a leptophobic $\mathrm{Z}^{\prime}$ boson and quarks [2] from various dijet analyses from CMS, ATLAS, CDF, and UA2. The limits are shown in solid lines, with the excluded area above the lines. The hashed areas show the direction of the excluded area from the observed limits. The grey dashed lines show the $g_{q}^{\prime}$ values at fixed values of $\Gamma_{Z^{\prime}} / M_{Z^{\prime}}$. Most of the analyses, with the exception of Dijet $\chi$ and Broad Dijet, assume that the intrinsic width is negligible compared to the experimental resolution, and hence are valid for $\Gamma_{Z^{\prime}} / M_{Z^{\prime}} \lesssim 10 \%$. The $t \bar{t}$ resonance analysis is valid for $\Gamma_{Z^{\prime}} / M_{Z^{\prime}} \lesssim 5 \%$, the Broad Dijet analysis is valid for $\Gamma_{Z^{\prime}} / M_{Z^{\prime}} \lesssim 30 \%$, and the Dijet $\chi$ analysis is valid for $\Gamma_{Z^{\prime}} / M_{Z^{\prime}} \lesssim 100 \%$. Also shown are indirect constraints on $g_{q}^{\prime}$ from the $\Upsilon$ and $Z$ boson widths, which are valid for all values of $\Gamma_{Z^{\prime}} / M_{Z^{\prime}}$.

These techniques can be compared in the context of a simplified model containing a leptophobic, spin-1 $Z^{\prime}$ boson, with equal vector couplings to quarks [2]. The Lagrangian for the model is:

$$
\mathscr{L} \ni g_{q}^{\prime} \sum_{i=1}^{6} \mathrm{Z}_{\mu}^{\prime} \bar{q}_{i} \gamma^{\mu} q_{i}
$$


Figure W shows limits on $g_{q}^{\prime}$ from UA2, CDF, ATLAS, and CMS, as well as indirect constraints from $\Upsilon$ decays and the hadronic width of the $Z$ boson [12]. The LHC results span three orders of magnitude in resonance mass, from $10 \mathrm{GeV}$ to $8 \mathrm{TeV}$. Proceeding from high masses to low, the traditional dijet search, using fully reconstructed events, probes masses above $\sim 1.5 \mathrm{TeV}$. With this method, the only cut with a significant impact on the signal acceptance is the requirement on pseudorapidity separation between the two jets, $\left|\Delta \eta_{j j}\right|<1$.1. Assuming that the resonance couples uniformly to all flavors of quarks, searches for $\bar{t} \bar{t}$ resonances have similar sensitivity in this range. For masses above $450 \mathrm{GeV}$, the traditional dijet methods can be applied to trigger-level events, where reduced event size allows for lower trigger thresholds. Between $250 \mathrm{GeV}$ and $450 \mathrm{GeV}$, the best limits are from searches targeting resonances produced with large ISR, reconstructed as two narrow, resolved jets. If we again assume that the resonance couples uniformly to all flavors of quarks, searches employing online b tagging have similar sensitivity in the range $325 \mathrm{GeV}-$ $450 \mathrm{GeV}$. Finally, for masses in the range $10 \mathrm{GeV}-250 \mathrm{GeV}$, searches for resonances with large transverse momentum due to ISR photons and jets have demonstrated unique sensitivity. In this phase space, the resonance decay products are collimated due to the large Lorentz boost, and thus are reconstructed as a single large-radius jet.

This report presents several new strategies developed during Run 2 by the CMS experiment which improve the sensitivity to lower resonance masses and smaller coupling values, pushing the mass coverage as low as $10 \mathrm{GeV}$. In particular, we describe two searches for dijet resonances produced with high transverse momentum due to significant initial state radiation [13, 14], as well as a new background estimation technique for the high mass search [15].

\section{The CMS Detector and Jet Reconstruction}

The central feature of the CMS apparatus is a superconducting solenoid of $6 \mathrm{~m}$ internal diameter, providing a magnetic field of 3.8 T. Within the solenoid volume are a silicon pixel and strip tracker, a lead tungstate crystal electromagnetic calorimeter (ECAL), and a brass and scintillator hadron calorimeter (HCAL), each composed of a barrel and two endcap sections. Forward calorimeters extend the pseudorapidity coverage provided by the barrel and endcap detectors. Muons are detected in gas-ionization chambers embedded in the steel flux-return yoke outside the solenoid. A more detailed description of the CMS detector, together with a definition of the coordinate system used and the relevant kinematic variables, can be found in Ref. [16].

The particle-flow (PF) algorithm [17] attempts to reconstruct and identify each individual particle in an event, with an optimized combination of information from the various elements of the CMS detector. The energy of photons is obtained from the ECAL measurement. The energy of electrons is determined from a combination of the electron momentum at the primary interaction vertex as determined by the tracker, the energy of the corresponding ECAL cluster, and the energy sum of all bremsstrahlung photons spatially compatible with originating from the electron track. The energy of muons is obtained from the curvature of the corresponding track. The energy of charged hadrons is determined from a combination of their momentum measured in the tracker and the matching ECAL and HCAL energy deposits, corrected for zero-suppression effects and for the response function of the calorimeters to hadronic showers. Finally, the energy of neutral hadrons is obtained from the corresponding corrected ECAL and HCAL energies. 
The analyses described here utilize jets formed from PF candidates. Narrow jets are reconstructed with the anti- $k_{\mathrm{T}}$ algorithm [18] with a distance parameter of $R=0.4$ (AK4). Wider jets, which aim to capture the decay products of massive particles with large Lorentz boost, are reconstructed with the anti- $k_{\mathrm{T}}$ algorithm with $R=0.8$ (AK8), or the Cambridge-Aachen algorithm with $R=1.5$ (CA15) [19]. The algorithms are implemented in the Fast Jet package [20]. The jet momentum is determined as the vectorial sum of all particle momenta in the jet, and is found from simulation to be, on average, within 5 to $10 \%$ of the true momentum over the whole $p_{\mathrm{T}}$ spectrum and detector acceptance. Jet energy corrections are derived from simulation studies so that the average measured response of jets becomes identical to that of particle level jets. In situ measurements of the momentum balance in dijet, photon+jet, Z+jet, and multijet events are used to determine any residual differences between the jet energy scale in data and in simulation, and appropriate corrections are made [21]. Contributions to the jet momentum from additional proton-proton interactions within the same or nearby bunch crossings are mitigated using the charged hadron subtraction (CHS) or pileup per particle identification (PUPPI) algorithms [22].

\section{High mass dijet search}

Proceeding from high resonance masses to low, we first present the search for dijet resonances at high mass, using $77.8 \mathrm{fb}^{-1}$ collected by the CMS detector [15]. The search targets narrow resonances decaying to two jets, with masses greater than $1800 \mathrm{GeV}$. This threshold is determined by the triggers used to collect events online, which require events to contain one or more jets with large transverse momenta. The analysis uses AK4 CHS jets. The two leading jets in $p_{\mathrm{T}}$ are chosen as the resonance decay candidates. To recover potential wide-angle radiation from the final-state partons, additional AK4 jets within a distance of $\Delta R<1.1$ of the leading two jets are added to the nearest leading jet. We refer to the resulting objects as "wide jets." The two resulting wide jets are each required to have $p_{\mathrm{T}}>30 \mathrm{GeV}$ and $|\eta|<2.5$, and the jet pair must satisfy $|\Delta \eta|<1.1$. The latter cut selects events with more central jets, and is imposed for two reasons: first, it improves the signal sensitivity, and second, it improves the efficiency of the trigger selection.

The background, dominantly due to QCD multijet events, is estimated in two ways. For $m_{j j}<$ $2.4 \mathrm{TeV}$, the background model is a smoothly falling, empirical function, which is fitted to the observed $m_{j j}$ spectrum ("fit method"). The function is given by:

$$
\frac{\mathrm{d} \sigma}{d m_{j j}}=\frac{P_{0}(1-x)^{P_{1}}}{x^{P_{2}+P_{3} \ln (x)}},
$$

where $x=m_{j j} / \sqrt{s}$.

For $m_{j j}>2.4 \mathrm{TeV}$, the background is estimated from a new technique using events with $|\Delta \eta|>$ 1.1 ("ratio method"). Three regions are defined: the signal region with $|\Delta \eta|<1.1$ (SR), a validation control region with $1.1<|\Delta \eta|<1.5\left(\mathrm{CR}_{\text {middle }}\right)$, and the background control region with $1.5<$ $|\Delta \eta|<2.5\left(\mathrm{CR}_{\text {high }}\right)$. The QCD background prediction in the signal region is taken from $\mathrm{CR}_{\text {high }}$, multiplied by a transfer factor from simulation: 


$$
\begin{aligned}
& N\left(m_{j j}\right)_{\mathrm{SR}}^{\text {Prediction }}=R_{\text {ext. }} \times N\left(m_{j j}\right)_{\mathrm{CR}_{\text {high }}^{\text {Data }}}^{\text {and }} \\
& R_{\text {ext. }}=\operatorname{Corr}\left(m_{j j}\right) \times \frac{N\left(m_{j j}\right)_{\mathrm{SR}}^{\text {Simulation }}}{N\left(m_{j j}\right)_{\mathrm{CR}_{\text {high }}}^{\text {Simulation }}} .
\end{aligned}
$$

The correction factor, parametrized as $\operatorname{Corr}\left(m_{j j}\right)=a+b\left(m_{j j} / \sqrt{s}\right)^{4}$, accounts for differences in the

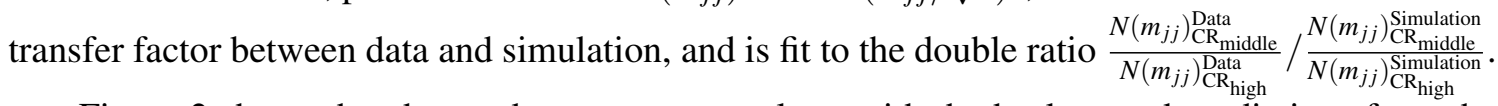

Figure $\square$ shows the observed $m_{j j}$ spectrum, along with the background predictions from the two methods and reference shapes for quark-quark, quark-gluon, and gluon-gluon signal models. No significant excess is observed. Upper limits on the product of the cross section $(\sigma)$, the branching fraction to dijets $(B)$, and the detector acceptance $(A)$ are shown in figure $[$ ]. The local significance of the data for quark-quark resonances is shown in figure $\mathbb{Q}$; note that the signficances for the ratio method are generally higher than those from the fit method, reflecting the reduced systematic uncertainty of the method.

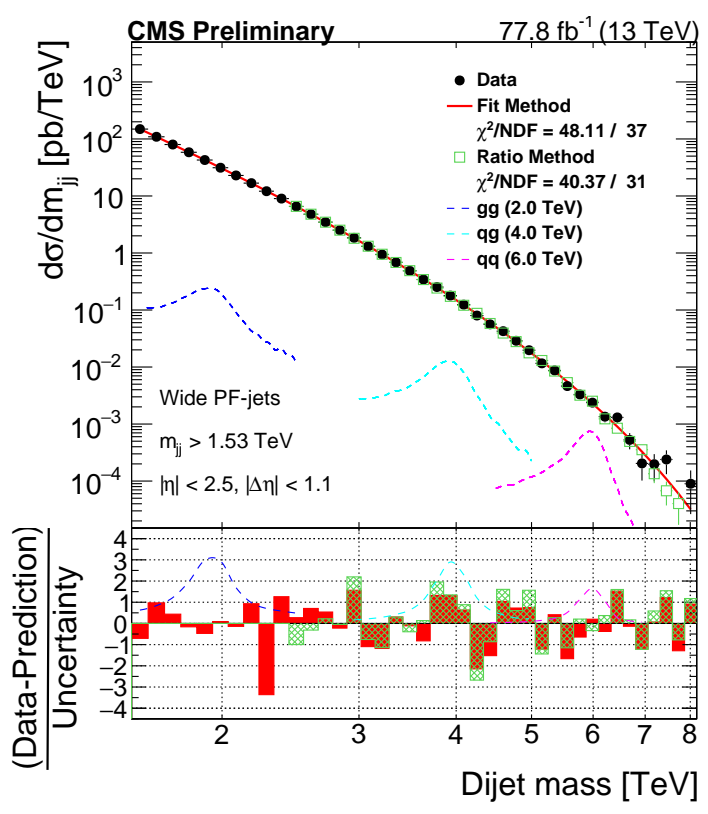

Figure 2: Dijet mass spectra in the signal region (points) compared to a fitted parameterization of the background (solid curve) and the one obtained from the control region (green squares). For the displayed signal a cross section at the $95 \%$ CL observed exclusion limit is being used. The lower panel shows the difference between the data and the fitted parametrization (red), and the data and the prediction obtained from the control region (green), divided by the statistical uncertainty of the data, which for the ratio method includes the one in $\mathrm{CR}_{\text {high }}$ as well. The ratio of the expected signal showed in the upper panel to the statistical uncertainty of the data is also shown for three different resonance masses and signals models. 

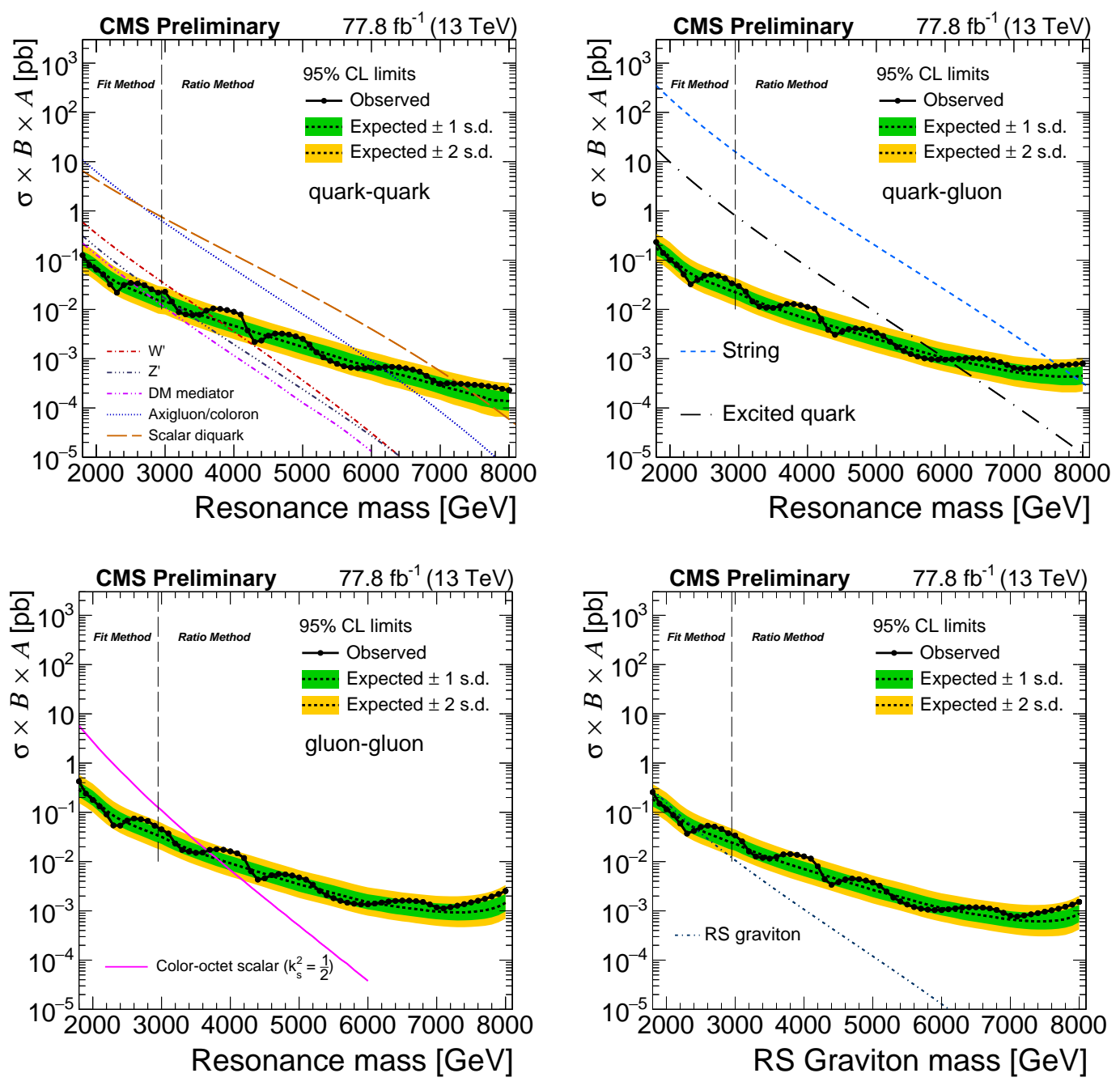

Figure 3: The observed 95\% CL upper limits on the product of the cross section, branching fraction, and acceptance for dijet resonances decaying to quark-quark (top left), quark-gluon (top right), gluon-gluon (bottom left), and for RS gravitons (bottom right). The corresponding expected limits (dashed) and their variations at the 1 and 2 standard deviation levels (shaded bands) are also shown. Limits are compared to predicted cross sections for string resonances, excited quarks, axigluons, colorons, scalar diquarks, color-octet scalars, new gauge bosons W' and Z' with SM-like couplings, dark matter mediators for $\mathrm{mDM}=1 \mathrm{GeV}$, and $\mathrm{RS}$ gravitons. 


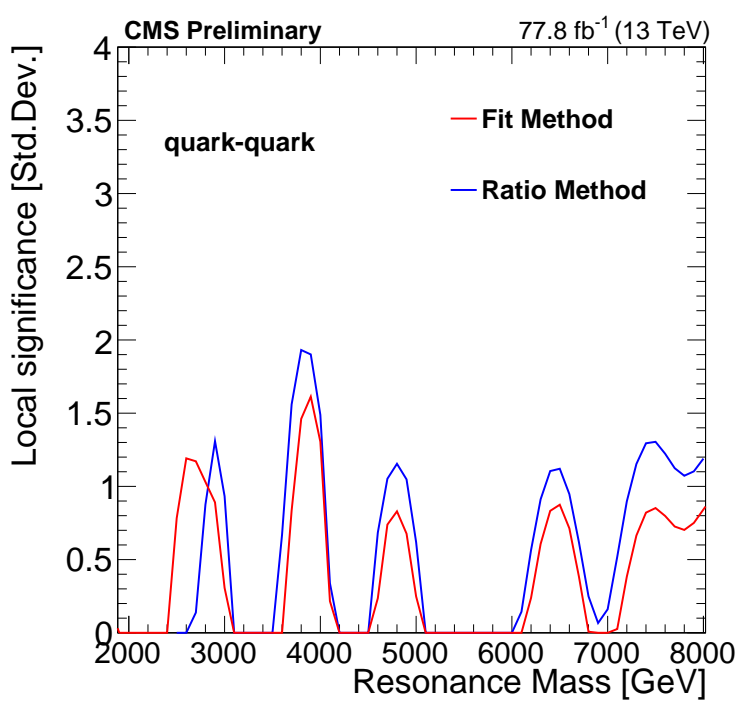

Figure 4: Local significance for a narrow resonance from the fit method (red) and the ratio method search (blue) for $q q$ resonances.

\section{Boosted dijet search with ISR jets}

The high-mass dijet resonance search probes resonance masses in the range $1800 \mathrm{GeV}-$ $8000 \mathrm{GeV}$; as mentioned previously, trigger-level analysis techniques allow the range to be extended down to about $450 \mathrm{GeV}$. To probe even lower masses, a more radical change in approach is needed to circumvent the trigger bandwidth limitations. The strategy which provides the best sensitivity in the range $10 \mathrm{GeV}-450 \mathrm{GeV}$ is to require that the resonance be produced with significant ISR $[14,23,24,25]$. We now present a search for $Z^{\prime}$ resonances produced with high transverse momentum due to ISR jets, using an integrated luminosity of $77.0 \mathrm{fb}^{-1}$ [13]. Due to the significant Lorentz boost, the resonance decay products are collimated into a single, large-radius jet. Using triggers that select events containing a large-radius jet with high mass and high transverse momentum, the search probes dijet resonance masses in the range $50 \mathrm{GeV}-450 \mathrm{GeV}$.

The search uses large-radius AK8 or CA15 jets built from PF candidates. The PUPPI algorithm is applied to reduce the impact of pileup interactions. AK8 jets, employed in both 2016 and 2017 data, are used for smaller resonance masses, where the decay products are more collimated. CA15 jets are only used in 2017 data, and improve the sensitivity at larger resonance masses. The triggers employ only AK8 jets, requiring that the event contain at least one jet with large trimmed mass [26], $m_{\text {trim }}>30 \mathrm{GeV}$, and untrimmed $p_{\mathrm{T}}>380 \mathrm{GeV}-400 \mathrm{GeV}$. The signal candidate is taken to be the AK8 or CA15 jet with the highest $p_{\mathrm{T}}$. The AK8 jets are required to have $p_{\mathrm{T}}>500 \mathrm{GeV}$ (2016) or $p_{\mathrm{T}}>525 \mathrm{GeV}$ (2017), due to different trigger thresholds in the two years. CA15 jets are required to have $p_{\mathrm{T}}>575 \mathrm{GeV}$. The jet must also satisfy $|\eta|<2.5$.

Jet substructure information is used to discriminate the signal from the dominant QCD background. The soft-drop mass algorithm [27], with $\beta=0$ and $z_{\text {cut }}=0.1$, removes soft and wide-angle radiation from the jet. The resulting soft-drop mass, $m_{\mathrm{SD}}$, tends to be significantly reduced for QCD jets, while the mass of jets due to $\mathrm{Z}^{\prime}$ candidates (as well as top quarks and $\mathrm{W}$ and $\mathrm{Z}$ bosons) 
is preserved. The $p_{\mathrm{T}}$-invariant variable $\rho \equiv \log \left(m_{\mathrm{SD}}^{2} / p_{\mathrm{T}}^{2}\right)$ is required to satisfy $-5.5<\rho<-2.0$ $(-4.7<\rho<-1.0)$ for AK8 (CA15) jets, to avoid non-perturbative effects at low mass and incomplete containment of the decay products within the jet cone at high masses. Finally, the observable $N_{2}^{1}$, based on 2- and 3-point energy correlation functions of the jet constituents, is used to select jets consistent with a two-pronged topology [28]. The variable is explicitly decorrelated from $m_{\mathrm{SD}}$ and $p_{\mathrm{T}}$ using the design decorrelated tagger method [29], defining:

$$
N_{2}^{1, \mathrm{DDT}}\left(\rho, p_{\mathrm{T}}\right) \equiv N_{2}^{1}\left(\rho, p_{\mathrm{T}}\right)-X_{(5 \%)}\left(\rho, p_{\mathrm{T}}\right),
$$

where $X_{(5 \%)}\left(\rho, p_{\mathrm{T}}\right)$ is the value of $N_{2}^{1}$ corresponding to $5 \%$ efficiency for the QCD background in a particular bin $\left(\rho, p_{\mathrm{T}}\right)$. Events with $N_{2}^{1, \mathrm{DDT}}<0$ constitute the signal, or "pass" region, while events with $N_{2}^{1, \text { DDT }}>0$ form the "fail" region. To improve the statistical precision of the computation of the quantiles, the method employs a gaussian kernel estimate: QCD events at generator level are "smeared" using a parametrization of the detector resolution in $N_{2}^{1}$ and $\rho$ as a function of $p_{\mathrm{T}}$ (accounting for correlations between the variables), multiplying the quantities at generator level by a random number taken from a Gaussian distribution so that the smeared distributions match those obtained from the full detector simulation.
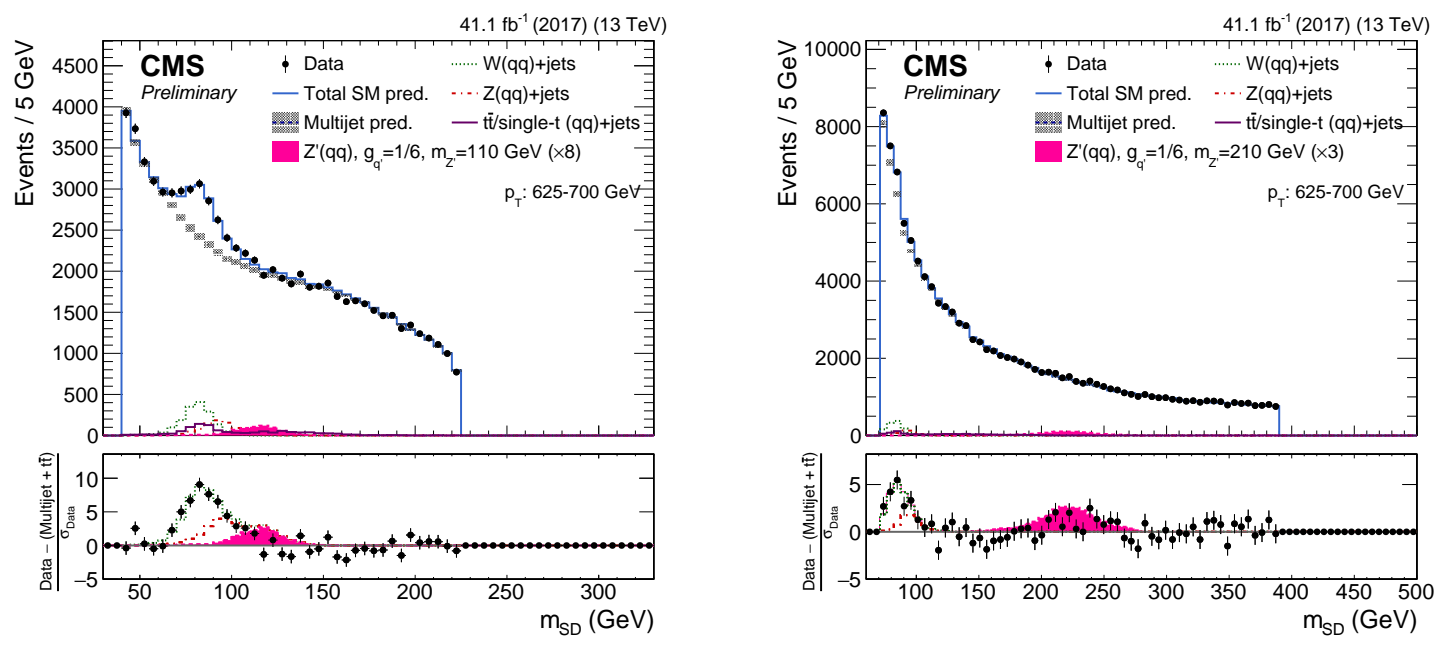

Figure 5: The $m_{\mathrm{SD}}$ distribution in data for AK8 (left) and CA15 (right) jets for the $625 \mathrm{GeV}-$ $700 \mathrm{GeV} p_{\mathrm{T}}$ category of the fit. Data are shown as black points. The multijet background prediction, including uncertainties, is shown by the shaded bands. Contributions from the $\mathrm{W}$ and $\mathrm{Z}$ bosons and the top quark background processes are shown. A hypothetical $Z^{\prime}$ boson signal with a mass of $110 \mathrm{GeV}$ and coupling $g_{q}^{\prime}=\frac{1}{6}$ is also indicated. The signal is scaled by a factor of 8 for clarity. In the bottom panel, the ratio of the data to its statistical uncertainty, after subtracting the nonresonant backgrounds, is shown. The signal is stacked on top of the peak formed by merged $\mathrm{W}$ and $\mathrm{Z}$ profiles.

The predictions of resonant backgrounds due to SM W and Z production are taken from simulation, as is that due to tz. The dominant QCD background is estimated from data events with $N_{2}^{1, \mathrm{DDT}}>0$. By construction, in simulation, the QCD mass and $p_{\mathrm{T}}$ distributions are the same in 
events with $N_{2}^{1, \text { DDT }}>0$ and $N_{2}^{1, \text { DDT }}<0$. To account for differences between data and simulation, the shape is multiplied by a polynomial transfer factor. Specifically, the QCD prediction is given by:

$$
n_{\text {pass }}^{\mathrm{QCD}}\left(\rho, p_{\mathrm{T}}\right)=R_{\mathrm{p} / \mathrm{f}}\left(\rho, p_{\mathrm{T}}\right) n_{\text {fail }}^{\mathrm{QCD}}\left(\rho, p_{\mathrm{T}}\right),
$$

where $R_{\mathrm{p} / \mathrm{f}}\left(\rho, p_{\mathrm{T}}\right)$ is a polynomial in $\rho$ and $p_{\mathrm{T}}$. The order of the polynomial is minimized using a Fisher F-test procedure, to limit the bias on the fitted signal strengths. A simultaneous fit is performed to all the $p_{\mathrm{T}}$ categories and the pass and fail regions, with the QCD shape in the fail region and the parameters of the polynomial left freely floating. The observed $m_{\mathrm{SD}}$ spectra for AK8 and CA15 jets in the transverse momentum range $625 \mathrm{GeV}-700 \mathrm{GeV}$ are shown in figure 5 , along with the predicted backgrounds and example signals. No significant excesses are observed, and limits on the coupling $g_{q}^{\prime}$ are shown in figure 6 . The jet type, AK8 or CA15, is chosen based on the expected sensitivity: CA15 jets are used above $225 \mathrm{GeV}$, while AK8 jets are used below.

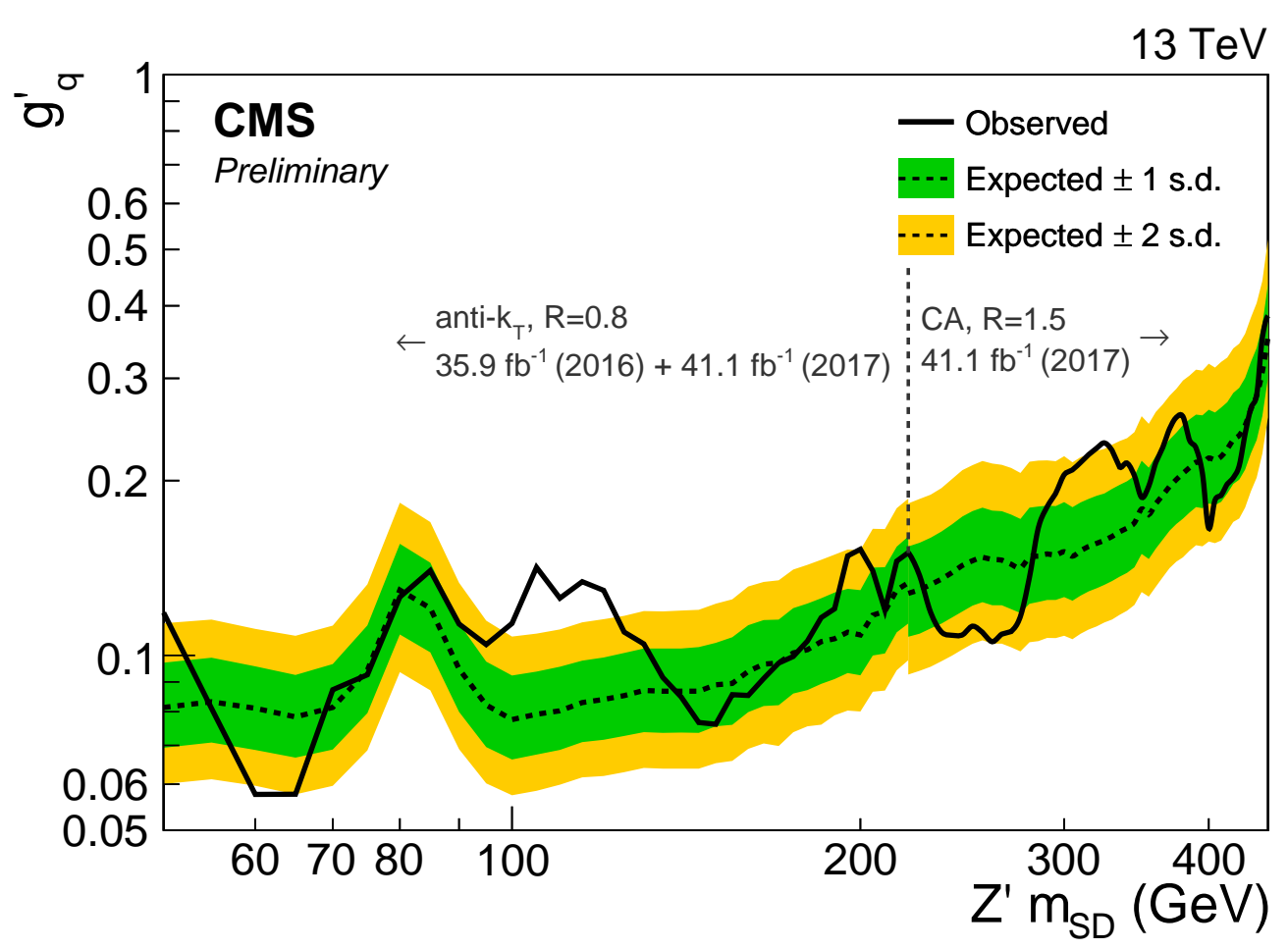

Figure 6: The upper limits at $95 \% \mathrm{CL}$ on the coupling $g_{q}^{\prime}$ as a function of resonance mass for a leptophobic $Z^{\prime}$ resonance that only couples to quarks. For masses between 50 and $220 \mathrm{GeV}$ the limits correspond to a $Z^{\prime}$ resonance reconstructed using the AK8 jet algorithm, using $77.0 \mathrm{fb}^{-1}$ of statistically combined data from 2016 and 2017. The excess in the observed limit over the expected limit near $120 \mathrm{GeV}$ is a remnant of the analysis of the data collected in 2016. For masses from $220 \mathrm{GeV}$ to $450 \mathrm{GeV}$, the limits correspond to a $\mathrm{Z}^{\prime}$ resonance reconstructed using the CA15 jet algorithm, using $41.1 \mathrm{fb}^{-1}$ of data collected in 2017 . 


\section{Boosted dijet search with ISR photons}

Using ISR jets to evade trigger limitations, the previously described search is able to probe dijet resonances with masses down to $50 \mathrm{GeV}$. The threshold can be lowered even further using ISR photons, which have a much lower $p_{\mathrm{T}}$ requirement in the trigger. We now describe a search for $\mathrm{Z}^{\prime}$ bosons produced with significant photon ISR, probing the mass range $10 \mathrm{GeV}-125 \mathrm{GeV}$ [14]. The search uses $35.9 \mathrm{fb}^{-1}$ of data collected in 2016 .

Events for the analysis are recorded using triggers that require a photon with $p_{\mathrm{T}}>175 \mathrm{GeV}$ and $|\eta|<3$.0. The analysis requires the photon to have $p_{\mathrm{T}}>200 \mathrm{GeV}$ and $|\eta|<2.4$, in order to have a constant trigger efficiency. Events with $|\eta|<2.1$ form the signal region, while events with $2.1<|\eta|<2.4$ are used as a control region for the $N_{2}^{1}$ decorrelation, described below. The analysis uses AK8 jets reconstructed from PF candidates, using the PUPPI algorithm to reduce the impact of pileup. The jet with the highest transverse momentum is chosen as the signal jet candidate, and is required to have $p_{\mathrm{T}}>200 \mathrm{GeV}$. The background contribution from $\mathrm{t} \overline{\mathrm{t}}$ is reduced by vetoing events with missing transverse momentum greater than $75 \mathrm{GeV}$, or containing an AK4 jet with $p_{\mathrm{T}}>$ $30 \mathrm{GeV}$ and satisfying the loose working point of the CSVv2 $b$ tagging algorithm [30]. Finally, the photon and the signal jet are required to be well-separated, satisfying $\sqrt{\Delta \eta^{2}+\Delta \phi^{2}}>2.2$.

As with the previous analysis, the soft-drop algorithm with $\beta=0$ and $z_{\text {cut }}=0.1$ is applied to the signal jet to reduce the mass of jets arising from quarks and gluons. The decorrelated $N_{2}^{1}$ variable is used to select jets with two-pronged substructure:

$$
N_{2}^{1, \mathrm{DDT}}\left(\rho, p_{\mathrm{T}}\right)=N_{2}^{1}\left(\rho, p_{\mathrm{T}}\right)-X_{(10 \%)}\left(\rho, p_{\mathrm{T}}\right) .
$$

Here, $X_{(10 \%)}\left(\rho, p_{\mathrm{T}}\right)$ is the $10^{\text {th }}$ percentile of $N_{2}^{1}$ in the corresponding $\left(\rho, p_{\mathrm{T}}\right)$ bin, computed using the control sample of events with photon pseudorapidity in the range $2.1<|\eta|<2.4$. A $K$-nearest neighbors smoothing algorithm is applied to the control sample to mitigate unphysical results in bins with limited statistics.

The backgrounds due to $\mathrm{SM} \mathrm{W}+\gamma, \mathrm{Z}+\gamma$, and $\mathrm{t} \overline{\mathrm{t}}$ are taken from simulation, as are the $\mathrm{Z}^{\prime}$ signal shapes. The QCD background is estimated using equation 4.2 , where $R_{\mathrm{p} / \mathrm{f}}$ now accounts for differences in the pass/fail ratio between the signal and control regions defined by the photon $\eta$.

A simultaneous fit is performed to the pass and fail $m_{\mathrm{SD}}$ distributions, with the QCD shape in the fail region and the coefficients of $R_{\mathrm{p} / \mathrm{f}}$ left freely floating. The observed $m_{\mathrm{SD}}$ distribution in the signal region is shown in figure $\square$, along with the predicted backgrounds and example signal distributions. No signficiant excess is observed, and limits are set on the coupling constant $g_{q}^{\prime}$ of the $Z^{\prime}$ model. The limits on $g_{q}^{\prime}$ are shown in figure 8 .

\section{Conclusion}

We have presented three searches for dijet resonances covering a broad range of resonance masses. For masses above $2400 \mathrm{GeV}$, we have introduced a new background estimation method which uses events in a sideband of jet pairs with large pseudorapidity separation. The method has reduced systematic uncertainty, and therefore better sensitivity, compared to the method based on empirical fits to the invariant mass spectrum commonly used to date. For masses below $450 \mathrm{GeV}$, where the event rate due to QCD multijet production exceeds the trigger bandwidth, searches for 


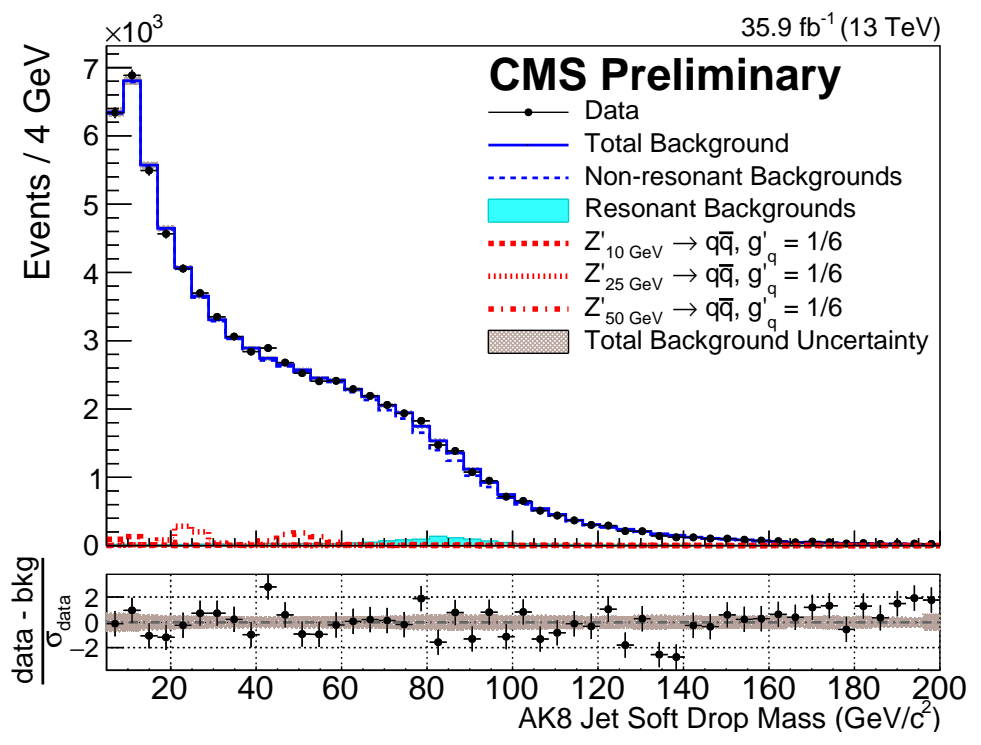

Figure 7: The soft drop jet mass distribution of the signal region after the main background estimation fit is performed. The nonresonant background is indicated by a dashed line, while the total background composed of the sum of this nonresonant background and the resonant backgrounds is shown by the solid line. Representative signals are plotted for comparison. The bottom panel shows the difference between the data and the final background estimate, divided by the statistical uncertainty of the data in each bin. The shaded region represents the total uncertainty in the background estimate in each bin.

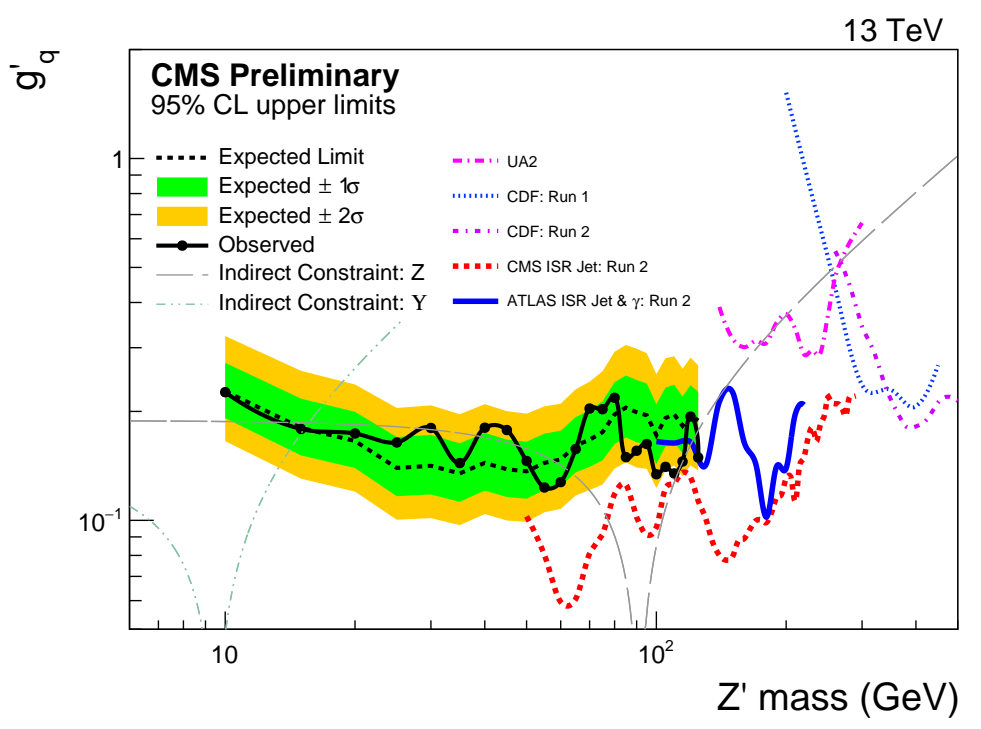

Figure 8: Upper limits at 95\% CL on the coupling strength $g_{q}^{\prime}$ of $\mathrm{Z}^{\prime} \rightarrow q \bar{q}$. The observed limit is shown as a solid black line, while the expected limit is dashed. The green (dark) and yellow (light) bands represent 1 and 2 standard deviation intervals. Limits from other searches and the indirect constraint from measurements of the $\Upsilon$ and $\mathrm{Z}$ boson decay widths are also shown. 
resonances produced with significant ISR have extended the mass range covered by LHC dijet searches down to $10 \mathrm{GeV}$. The two searches presented, targeting resonances produced with either an ISR photon or an ISR jet, have demonstrated the best sensitivity in the mass range $10 \mathrm{GeV}-$ $50 \mathrm{GeV}$ and $10 \mathrm{GeV}-50 \mathrm{GeV}$ and $50 \mathrm{GeV}-250 \mathrm{GeV}$, respectively.

\section{References}

[1] E. Eichten et al. "Supercollider physics". In: Rev. Mod. Phys. 56 (4 Oct. 1984), pp. 579-707. DOI: 10.1103/RevModPhys.56.579. URL: https:// link.aps.org/doi/10. 1103 /RevModPhys. 56.579.

[2] Bogdan A. Dobrescu and Felix Yu. "Coupling-mass mapping of dijet peak searches". In: Phys. Rev. D 88 (3 Aug. 2013), p. 035021. DOI: 10.1103 / PhysRevd . 88 . 035021. URL: https://link.aps.org/doi/10.1103/PhysRevD.88.035021.

[3] U. Baur, I. Hinchliffe, and D. Zeppenfeld. "Excited Quark Production at Hadron Colliders". In: Int. J. Mod. Phys. A2 (1987), p. 1285. DOI: $10.1142 /$ S $0217751 \times 87000661$.

[4] U. Baur, M. Spira, and P. M. Zerwas. "Excited-quark and -lepton production at hadron colliders”. In: Phys. Rev. D 42 (3 Aug. 1990), pp. 815-824. DOI: 10.1103 / Phys RevD . 42. 815. URL: https://link.aps.org/doi/10.1103/PhysRevD . 42.815.

[5] Lisa Randall and Raman Sundrum. "An Alternative to Compactification". In: Phys. Rev. Lett. 83 (23 Dec. 1999), pp. 4690-4693. DoI: 10.1103 / PhysRevLett . 83 . 4690. URL: https://link.aps.org/doi/10.1103/PhysRevLett.83.4690.

[6] Robert M. Harris and Konstantinos Kousouris. "Searches for Dijet Resonances at Hadron Colliders". In: Int. J. Mod. Phys. A26 (2011), pp. 5005-5055. DOI: 10 . 1142 / S 0217751 X11 054905. arXiv: 1110.5302 [hep-ex].

[7] CMS Collaboration. "Search for narrow and broad dijet resonances in proton-proton collisions at $\sqrt{s}=13 \mathrm{TeV}$ and constraints on dark matter mediators and other new particles". In: JHEP 08 (2018), p. 130. DOI: 10.1007 / JHEP 08 (2018) 130. arXiv: 1806.00843 [hep-ex].

[8] Morad Aaboud et al. "Search for new phenomena in dijet events using $37 \mathrm{fb}^{-1}$ of $p p$ collision data collected at $\sqrt{s}=13 \mathrm{TeV}$ with the ATLAS detector". In: Phys. Rev. D96.5 (2017), p. 052004. DOI: 10.1103/PhysRevD.96.052004. arXiv: 1703.09127 [hep-ex] .

[9] Vardan Khachatryan et al. "Search for narrow resonances in dijet final states at $\sqrt{(s)}=8 \mathrm{TeV}$ with the novel CMS technique of data scouting". In: Phys. Rev. Lett. 117.3 (2016), p. 031802. DOI: 10.1103/PhysRevLett.117.031802. arXiv: 1604.08907 [hep-ex] .

[10] M. Aaboud et al. "Search for low-mass dijet resonances using trigger-level jets with the ATLAS detector in $p p$ collisions at $\sqrt{s}=13$ TeV". In: Phys. Rev. Lett. 121.8 (2018), p. 081801. DOI: 10.1103/PhysRevLett.121.081801. arXiv: 1804.03496 [hep-ex] .

[11] CMS Collaboration. "Search for narrow resonances in the b-tagged dijet mass spectrum in proton-proton collisions at $\sqrt{s}=8 \mathrm{TeV}$ ". In: Phys. Rev. Lett. 120.20 (2018), p. 201801. DOI: 10.1103/PhysRevLett.120.201801. arXiv: 1802.06149 [hep-ex] . 
[12] Bogdan A. Dobrescu and Claudia Frugiuele. "Hidden GeV-scale interactions of quarks". In: Phys. Rev. Lett. 113 (2014), p. 061801. DOI: 10.1103 / PhysRevLett. 113.061801. arXiv: 1404.3947 [hep-ph] .

[13] CMS Collaboration. "Search for low mass vector resonances decaying into quark-antiquark pairs in proton-proton collisions at $\sqrt{s}=13 \mathrm{TeV}$ ". In: (2019). arXiv: 1909.04114 [hep-ex] .

[14] CMS Collaboration. "Search for low-mass quark-antiquark resonances produced in association with a photon at $\sqrt{s}=13 \mathrm{TeV}$ ". In: (2019). arXiv: 1905.10331 [hep-ex] .

[15] Searches for dijet resonances in pp collisions at $\sqrt{s}=13 \mathrm{TeV}$ using the 2016 and 2017 datasets. Tech. rep. CMS-PAS-EXO-17-026. Geneva: CERN, 2018. uRL: http: / / cds . cern.ch/record/2637847.

[16] CMS Collaboration. "The CMS experiment at the CERN LHC". In: Journal of Instrumentation 3.08 (Aug. 2008), S08004-S08004. DOI: 10 .1088/1748-0221/3/08/s 08004. URL: https://doi.org/10.1088/1748-0221/3/08/s08004.

[17] CMS Collaboration. "Particle-flow reconstruction and global event description with the CMS detector”. In: JINST 12.10 (2017), P10003. DOI: 10 . 1088/1748-0221/12/10/ P10003. arXiv: 1706.04965 [physics.ins-det] .

[18] Matteo Cacciari, Gavin P Salam, and Gregory Soyez. "The anti-ktjet clustering algorithm". In: Journal of High Energy Physics 2008.04 (Apr. 2008), pp. 063-063. DOI: 10 . 1088 / 1126-6708/2008/04/063. uRL: https : / / doi . org/10 . 1088/1126$6708 / 2008 / 04 / 063$.

[19] Yu.L Dokshitzer et al. "Better jet clustering algorithms". In: Journal of High Energy Physics 1997.08 (Aug. 1997), pp. 001-001. DOI: $10.1088 / 1126-6708 / 1997 / 08 / 001$. URL: https://doi.org/10.1088/1126-6708/1997/08/001.

[20] Matteo Cacciari, Gavin P. Salam, and Gregory Soyez. "FastJet user manual". In: The European Physical Journal C 72.3 (Mar. 16, 2012), p. 1896. ISSN: 1434-6052. DOI: 10.1140 / epjc/s10052-012-1896-2. URL: https : / / doi .org/10.1140/epjc/ s10052-012-1896-2.

[21] "Jet energy scale and resolution performance with $13 \mathrm{TeV}$ data collected by CMS in 2016". In: (June 2018). URL: http: / / cds . cern. ch/record/2622157.

[22] CMS Collaboration. "Pileup mitigation at CMS in $13 \mathrm{TeV}$ data". In: (2019).

[23] CMS Collaboration. "Search for low mass vector resonances decaying into quark-antiquark pairs in proton-proton collisions at $\sqrt{s}=13 \mathrm{TeV}$ ". In: JHEP 01 (2018), p. 097. DOI: 10 . 1007 / JHEP 01 (2018) 097. arXiv: 1710.00159 [hep-ex].

[24] ATLAS Collaboration. "Search for light resonances decaying to boosted quark pairs and produced in association with a photon or a jet in protonproton collisions at $\mathrm{s}=13 \mathrm{TeV}$ with the ATLAS detector". In: Physics Letters B 788 (2019), pp. 316-335. ISSN: 0370-2693. DOI: https: / / doi.org/10.1016/j.physletb.2018.09.062. URL: http: / / www.sciencedirect.com/science/article/pii/s037026931830830X. 
[25] ATLAS Collaboration. "Search for low-mass resonances decaying into two jets and produced in association with a photon using pp collisions at $\mathrm{s}=13 \mathrm{TeV}$ with the ATLAS detector". In: Physics Letters B 795 (2019), pp. 56-75. ISSN: 0370-2693. DOI: https : // doi.org/10.1016/j.physletb.2019.03.067. URL: http: / / www . sciencedirect.com/science/article/pii/s0370269319303612.

[26] David Krohn, Jesse Thaler, and Lian-Tao Wang. "Jet Trimming”. In: JHEP 02 (2010), p. 084. DOI: 10.1007 / JHEP 02 (2010) 084. arXiv: 0912.1342 [hep-ph] .

[27] Andrew J. Larkoski et al. "Soft Drop”. In: JHEP 05 (2014), p. 146. DOI: 10 . 1007 / JHEP 05 (2014) 146. arXiv: 1402.2657 [hep-ph] .

[28] Ian Moult, Lina Necib, and Jesse Thaler. "New Angles on Energy Correlation Functions". In: JHEP 12 (2016), p. 153. DOI: 10.1007 / JHEP 12 (2016) 153. arXiv: 1609.07483 [hep-ph].

[29] James Dolen et al. "Thinking outside the ROCs: Designing Decorrelated Taggers (DDT) for jet substructure”. In: JHEP 05 (2016), p. 156. DOI: 10 . 1007 / JHEP 05 (2016) 156. arXiv: 1603.00027 [hep-ph].

[30] CMS Collaboration. "Identification of heavy-flavour jets with the CMS detector in pp collisions at 13 TeV”. In: JINST 13.05 (2018), P05011. DOI: 10 . 1088/1748-0221/13/ 05/P05011. arXiv: 1712.07158 [physics.ins-det]. 